Received: 2013.05.30 Accepted: 2013.07.01 Published: 2013.11.18

Authors' Contribution: Study Design A Data Collection B Statistical Analysis C Data Interpretation D Manuscript Preparation E Literature Search F Funds Collection $G$

\author{
ABDG Heidar Dadkhah-Tirani \\ ABG Arsalan Salari \\ CDEFG Shora Shafighnia \\ BDG Seyed Fazel Hosseini \\ CDFG Misa Naghdipoor
}

Department of Cardiology, Dr. Heshmet Hospital, Guilan University of Medical Sciences, Rasht, Iran

\title{
Coronary artery to pulmonary artery fistula
}

\author{
Patient: Male, 69 \\ Final Diagnosis: \\ Symptoms: \\ Coronary artery to pulmonary artery fistula \\ Medication: \\ Chest pain \\ Clinical Procedure: \\ Echocardiography • angiography • surgical intervention \\ Specialty: \\ Cardiology • Cardiac Surgery \\ Objective: \\ Rare disease \\ Background: \\ A coronary artery fistula is an abnormal communication between a coronary artery and one of the cardiac cham- \\ bers or a great vessel, so bypassing the myocardial capillary network. They are usually discovered incidentally \\ upon coronary angiography. Clinical manifestations are variable depending on the type of fistula, the severity \\ of shunt, site of shunt, and presence of other cardiac condition. \\ Case Report: We report a 69-year-old man without any previous medical history, who was admitted to our hospital with \\ chest pain. The electrocardiogram (ECG) showed a sinus rhythm with ST depression in V2 to V6 precordial leads. \\ Coronary angiography revealed a coronary artery fistula from left anterior descending coronary artery (LAD) to \\ the main pulmonary artery, right coronary artery blockage and significant stenoses on the LAD and left circum- \\ flex artery (LCX). \\ Conclusions: Surgical treatment was chosen because of the total occlusion of the right coronary artery and to relieve of pain \\ to improve quality of life. \\ Key words: left anterior descending coronary artery • electrocardiogram • coronary artery • fistula • angiography \\ - pulmonary artery • left circumflex artery
}

Full-text PDF: http://www.amjcaserep.com/download/index/idArt/889416

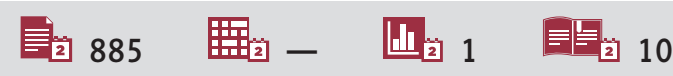




\section{Background}

Coronary artery fistulas (CAF) are uncommon vascular communications between a coronary artery and one of the cardiac chambers or a great vessel around heart,so lead to bypass the myocardial capillary network [1-3]. Coronary artery fistulae represent the most frequent congenital anomalies of the coronary arteries, but remain a relatively uncommon clinical problem [4]. These fistulas are usually discovered incidentally upon coronary angiography [2,3]. Various symptoms and signs related to the fistula depending on the type of fistula, shunt volume, site of shunt, and presence of other cardiac conditions $[1,2]$. The majority of these fistulas are congenital but can nevertheless arise after a cardiac surgery [4]. Their incidence in the overall population is reported about $0.002 \%$ [1-3] and constitute $0.13 \%$ of congenital cardiac lesion [1,3] however, they are found in $0.05 \%$ to $0.25 \%$ of patients who undergo coronary angiography [5]. The most common site of drainage is the right ventricle (41\%) [5]. Drainage of the fistula into the pulmonary trunk has been reported in $17 \%$ of cases $[3,5]$. The operation is indicated when clinical symptom occur, such as cardiac insufficiency and myocardial ischemia, or the presence of a high flux fistula in an asymptomatic patient [6].

\section{Case Report}

A 69 year-old man (weight $67 \mathrm{~kg}$, height $1.64 \mathrm{~cm}$ ) was referred to cardiology clinic of Guilan with chest pain. His chest pain was retrosternal and effort-related, was relieved by rest, radiated to left arm, which was associated with diaphoresis. He had no history of diabetic mellitus, hypertension, hyperlipidemia, smoking and family history of coronary artery disease. There were no signs of cardiopulmonary insufficiency. Physical examination and heart auscultation revealed nothing unusual. The jugular venous pressure was not elevated and ECG showed a sinus rhythm of 76 beats/min, with ST depression in V2 to V6 precordial leads. The transthoracic echocardiogram demonstrated normal wall motion with an ejection fraction of $55 \%$ and heart function valve was unremarkable. Afterward, the patient became a candidate for coronary angiography. He underwent a coronary arteriogram a week later, which revealed a fistula connection arising from the LAD and draining into the main pulmonary artery, blockage of the right coronary artery and significant stenoses on the LAD and LCX (Figure 1A, 1B). Surgical treatment was chosen because of the total occlusion of the right coronary artery and to relieve pain to improve quality of life.

\section{Surgical technique}

Following general anesthesia, a median sternotomy, and longitudinal opening of the pericardium, the anatomy of coronary artery was evaluated. A fistula was identified at the origin of the

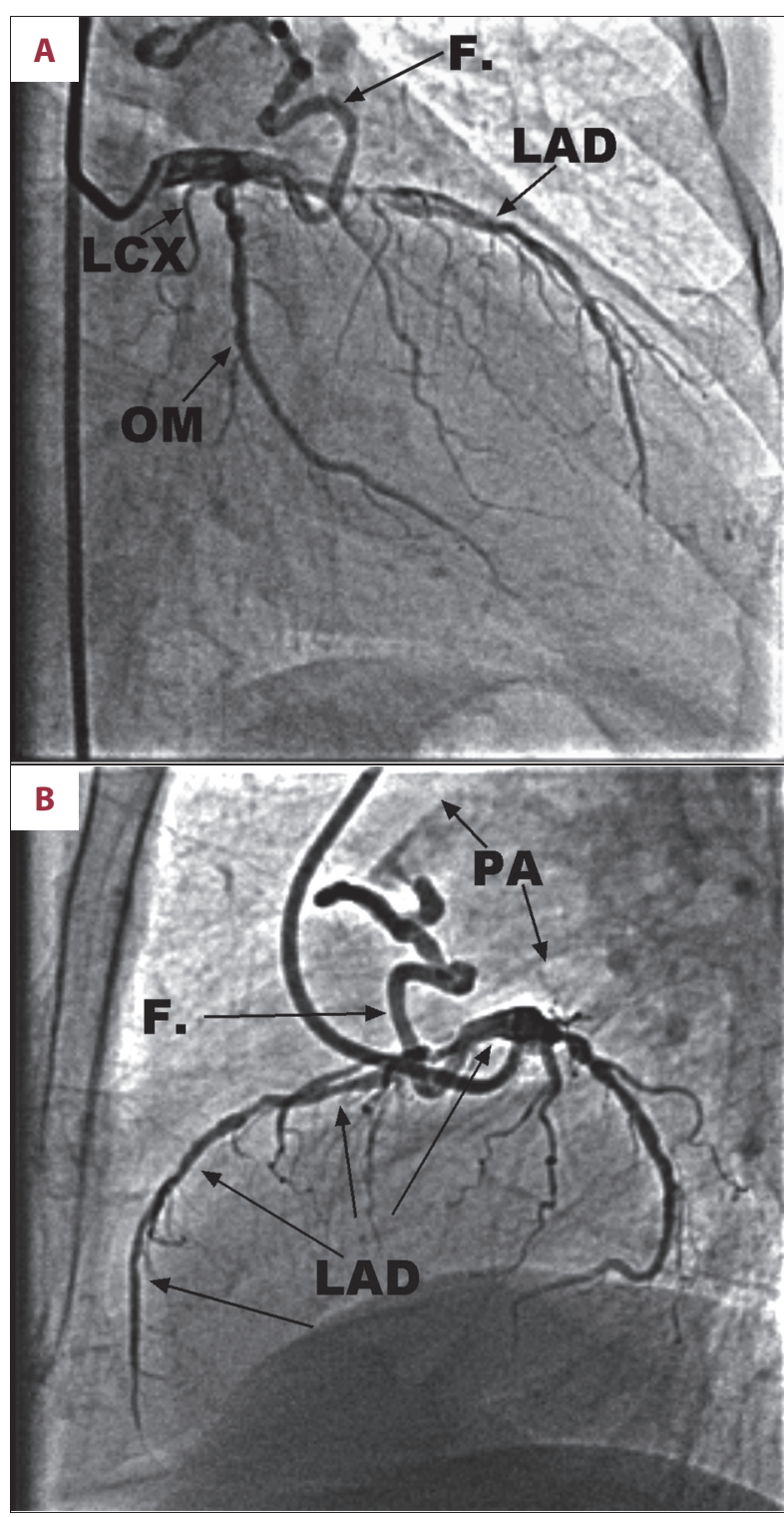

Figure 1. (A) Coronary angiography confirms that fistula between left anterior descending coronary artery (LAD) and main Pulmonary Artery (PA), also, stenoses on the LAD and left circumflex artery (LCX). (B) Fistula which located between left anterior descending coronary artery $(\mathrm{LAD})$ and main pulmonary artery (PA).

proximal left anterior descending artery (LAD), which drained into the main pulmonary artery. The operation was performed with the aid of cardiopulmonary bypass, moderate hypothermia, and intermittent combined (retro and anterograde) cold blood cardioplegia.

The orifice of the fistula was closed with 4-0 prolene via pulmonary arteritomy. Afterward, triple-vessel coronary artery bypass grafting was performed. During the operation, no hemodynamic instability was observed. The patient left the ICU 2 
days after surgery and left the hospital 8 days after surgery. After the operation, the patient was symptom free. A nuclear scan performed 4 months later showed normal myocardial perfusion, and no residual fistula.

\section{Discussion}

Coronary artery fistulas are very rare congenital anomalies which and constitute 0.2 to $0.4 \%$ of all cases of congenital heart diseases. In a review of 363 cases of CAF, $50 \%$ of the fistulas were found to arise from the RCA, $42 \%$ from the left coronary artery, and $5 \%$ from both coronary arteries. The most common site of drainage is the right ventricle (41\%), followed by the right atrium (26\%) and the pulmonary artery (17\%) [1]. A fistula between the left anterior descending artery and the main pulmonary artery, as in the case, is a very rare finding [3,7]. The main indications for closure of CAF are clinical symptoms, particularly heart failure and myocardial ischemia and, in asymptomatic patients with high-flow shunting, to prevent occurrence of undesirable complications [2]. Surgical intervention to correct the fistula is recommended for symptomatic patients and for those asymptomatic patients at risk for future complications such as bacterial endocarditis, thrombosis, distal embolization, aneurysm, dissection, rupture, premature atherosclerosis, pulmonary hypertension, myocardial ischemia or infarction $[2,8,9]$.

\section{References:}

1. Papadopoulos DP, Perakis A, Votreas V, Anagnostopoulou S: Bilateral fistulas: a rare cause of chest pain. Case report with literature review. Hellenic J Cardiol, 2008; 49: 111-13

2. Jerbi S, Tarmiz A, Fradi S et al: Coronary artery fistula: case report and review of the literature. Ann Cardiol Angeiol (Paris), 2009; 58: 236-39

3. Papadopoulos DP, Bourantas CV, Ekonomou CK, Votteas V: Coexistence of atherosclerosis and fistula as a cause of angina pectoris: a case report. Cases J, 2010; 3: 70

4. Kwon SH, Oh JH, Hwang S-J et al: Bilateral Coronary Artery Fistulae to Main Pulmonary Artery Discovered Incidentally upon Multidetector-Row Computed Tomography. Tex Heart Inst J, 2010; 37(1): 127-28

5. Iglesias JF, Thai HT, Kabir T et al: Transcatheter coil embolization of multiple bilateral congenital coronary artery fistulae. J Invasive Cardiol, 2010 22: $142-45$
The increased experience and the improved devices and techniques provide a variety of therapeutic options. The preferred approach for any individual patient will depend on the anatomy of the fistula, the presence or absence of associated defects, and the experience of the interventional cardiologists and surgeons. In fact, the optimal management of symptomatic patients with coronary artery fistulae still remains controversial. However, results from the transcatheter and surgical literature show that both approaches have similar early effectiveness, morbidity, and mortality. Surgical intervention (coronary artery bypass surgery and CAF closure) was chosen for treating this patient due to the total obstruction of the right coronary artery in combination with a CAF. In a similar case, reported by Papadopoulos et al., surgery was performed. Hence, we believe that surgical closure of CAF offers extraordinary results and in similar cases should be the firstline treatment choice.

\section{Conclusions}

Our case is a good example of a rare congenital anomaly in which coronary artery blockage can remain entirely asymptomatic over many years. Despite the fact that CAF is rare, this diagnosis should be considered in all patients who present with angina and exertional dyspnea, as was evident in this case.

6. Guedes MA, Riso Ade A, Barbero Marcial M, Stolf NA: Off-pump correc tion of coronary fistula: the single suture technique approach. Rev Bras Cir Cardiovasc, 2009; 24: 581-83

7. Tacoy G, Ebinc H, Onal B et al: Congenitally severe tortuous circumflex artery fistula draining into the coronary sinus: Transcatheter closure with Guglielmi detachable coils via different delivery system. J Cardiol, 2009; 54: 317-21

8. Ekonomou CK, Papadopoulos DP, Dalianis NV et al: Coronary fistula from left main stem to main pulmonary artery. J Invasive Cardiol, 2003, 15: 600-1

9. Luo L, Kebede S, Wu S, Stouffer GA: Coronary artery fistulae. Am J Med Sci, 2006; 332: 79-84

10. Koneru J, Samuel A, Joshi M et al: Coronary Anomaly and Coronary Artery fistula as cause of Angina Pectoris with Literature Review. Case Rep Vasc Med, 2011; 2011: 486187 